\title{
Radiation dose reduction without degrading image quality during computed tomography examinations: Dosimetry and quality control study
}

\author{
George Felix Acquah", Bernhard Schiestt ${ }^{1,2}$, Afua Yeboah Cofie ${ }^{1}$, Jeanette Obeng Nkansah ${ }^{1}$, \\ Magnus Gustavsson ${ }^{3}$ \\ ${ }^{1}$ Department of Radiation Oncology, Sweden Ghana Medical Centre, Cantonments, Accra, Ghana. \\ ${ }^{2}$ Department of Therapeutic Radio Oncology, University Hospital of Innsbruck, Innsbruck, Austria. \\ ${ }^{3}$ Department of Medical Physics, Sahlgrenska University Hospital, Gothenburg, Sweden.
}

Received March 11, 2014; Revised May 12, 2014; Accepted May 18, 2014; Published Online June 04, 2014

\section{Technical Report}

\begin{abstract}
Purpose: Computed tomography (CT), is an X-ray procedure that generates high quality cross-sectional images of the body, and by comparison to other radiological diagnosis, is responsible for higher doses to patients. This work studies the doses and image qualities produced from the default primary scanning factors of a Siemens CT machine and afterwards came up with scanning protocols that allow radiologists to obtain the necessary diagnostic information while reducing radiation doses to as low as reasonably achievable. Methods: Approximately 1000 CT scans from mostly common examinations; head, thorax, abdomen and pelvis routines were selected and analyzed for their image quality and radiation doses over a two year interval. Dose measurements were performed for the same routines using Computed Tomography Dose Index (CTDI) phantoms, RTI barracuda system with electrometer, and CT dose Profiler detector to evaluate the doses delivered during these CT procedures. Subsequently, image quality checks were performed using the CT Catphan 600 and anthropomorphic phantoms. CTDI and Dose Length Product (DLP) values were calculated for each scan. From analyzing these measurements, the appropriate machine scanning parameters were adjusted to reduce radiation does while at the same time providing good image quality. Results: Doses to patients using the default head sequence protocol had an average CTDIvol value of $65.45 \mathrm{mGy}$ and a range of 7.10-16.80 mGy for thorax, abdomen and pelvis examinations whiles the new protocol had an average CTDIvol of $58.32 \mathrm{mGy}$ for the head and a range of 3.83-15.24 mGy for the truck region. The DLP value for default head scans decreased from an average of 2279.85 mGy.cm to $874.53 \mathrm{mGy} . \mathrm{cm}$ with the new protocol. Tube potentials (KV) and tube current-time (mAs) had an effect on spatial resolution and low contrast detectability as well as doses. Conclusion: From the new protocols, lower values of KV and $\mathrm{mAs}$ together with other factors were enough to produce acceptable level of image quality which leads to adequate diagnosis without unnecessary doses to patients.
\end{abstract}

Keywords: Computerized Tomography; Radiation Dose Reduction; Image Quality; CTDI; DLP

\section{Introduction}

Computed tomography is one of the most frequently used diagnostic imaging methods. Despite the universal consensus that CT benefits patients when used appropriately, concerns

Corresponding author: George Felix Acquah; Department of Radiation Oncology, Sweden Ghana Medical Centre, Cantonments, Accra, Ghana.

Cite this article as:

Acquah GF, Schiestl B,Cofie AY, Nkansah JO, Gustavsson M. Radiation dose reduction without degrading image quality during computed tomography examinations: Dosimetry and quality control study. Int J Cancer Ther Oncol 2014; 2(3):02039.

DOI: $10.14319 /$ ijcto.0203.9 have been raised regarding potential risk of cancer induction from over exposure. Recent overdose incidents led to new interest in evaluating the dose delivered during CT exams. ${ }^{1}$ Keeping radiation dose as low as reasonably achievable, consistent with the diagnostic task, remains the most important strategy for decreasing this potential risk of radiation-induced malignancy. ${ }^{2}$ Many somatic effects of radiation become evident a few months after use of the X-ray in diagnostic medical applications. In requesting CT examination, two guiding principles must be followed. ${ }^{3}$ First, CT examinations must be appropriately justified for each individual patient. ${ }^{4}$ Second, 
for each CT examination, all technical aspects of the examination must be optimized, such that the required levels of image quality can be obtained while keeping the doses low. This article focuses on the second guiding principle and discusses the technical strategies adopted for radiation dose reduction. Radiation dose can be quantified by scanner radiation output, organ dose, effective dose etc. The scanner radiation output is represented by the volume CTDI $\mathrm{vol}_{\mathrm{l}}$, which describes the radiation output of the scanner by using the head and body CTDI phantoms. The radiation doses delivered to patients during medical examinations as well as workers are of interest from a radiation protection point of view. Also an assessment of the radiation risk may be based on one or two specific organs doses, or the effective dose, as recommended by the International Commission for Radiological Protection (ICRP). ${ }^{5}$ Regular quality assurance measurements on CT scanners are necessary in order to monitor the dose levels patients are exposed to during medical examinations. In many countries, governments require regular quality compliance testing information from clinics and hospitals that perform CT examinations.

However, as radiation-related diagnosis and treatment techniques become more sophisticated, patients are more likely to be subject to radiation exposure that is too risky to ignore. While not overriding the benefits gained from the procedures, it is highly desirable to develop techniques to reduce patient dose without impacting the quality of care. According to the United Nations Scientific Committee on the Effects of Atomic Radiation (UNSCEAR), worldwide, CT constitutes approximately $6 \%$ of all medical X-ray examinations; its contribution to the resultant collective dose was about $41 \%$ in 1999-2000. ${ }^{6}$ Due to the increase use of CT and added applications, the CT contribution to collective dose is growing over the decades. In the UK, CT scan contributions has doubled over 10 years to about $47 \%{ }^{7}$ representing about $9 \%$ of all X-ray examinations. ${ }^{8}$

Effective dose calculation is the best available predictor of the stochastic risk of a given radiological examination as it estimates or calculates the dose of organs. ${ }^{9}$ Study by Wade JP et al ${ }^{10}$ concluded that, the effective dose for the head scan is considerably less than that for the trunk scans, even though the CTDI values for head scan are much higher. This is because fewer of the radiosensitive organs are irradiated. Actually, the effective dose value is a reflection of the overall factors that determine the radiation exposure produced by the machine. Those factors include design characteristics for each scanner, (for example the focus-to-axis distance), and the physical factors selected for each exam, such as the $\mathrm{KV}$, $\mathrm{mAs}$, slice thickness and number of slices. It was observed in this study that in general the effective dose values are correlated to the corresponding dose length product values, i.e. low DLP values leads to low effective dose values. The method used to evaluate these doses is the computed tomography dose index, which represents the absorbed dose along the longitudinal axis (z-axis) of the CT scanner measured during a single rotation of the X-ray source. CTDI is commonly measured with along pencil ionization chamber placed in a phantom representing adult head and body. ${ }^{11}$ It was found that using the standard $10 \mathrm{~cm}$ long pencil ionization chamber placed in a 14 cm long Polymethyl methacrylate (PMMA) phantom may result in inaccurate measurements due to its tendency to underestimate the dose profile. And for the new generation of CT scanners, the efficiency of this methodology is low because it excludes the contribution of radiation scattered beyond the $100 \mathrm{~mm}$ range of integration along the longitudinal (z) axis. ${ }^{12}$ The answer to this problem is the CT Dose Profiler (CTDP) probe.

CTDIvol and its related quantities, such as weighted CTDI and dose length product, are widely used for quality assurance testing and to describe and optimize the radiation output. These are not direct measurements of patient dose; they are standardized dose metric to represent scanner output levels, when measured in a standardized phantom. Diagnostic reference levels do not indicate the desired dose level for a specific diagnostic task, but rather define a reference dose, above which users should investigate the potential dose-reduction measures. Diagnostic reference levels have been established by the European Commission (EC) and several of its member state, as well as the American College of Radiology (ACR), for adult head, adult abdomen and pediatric abdomen. ${ }^{13,14}$ Radiation dose should only be reduced under the condition that the diagnostic image quality is not sacrificed. Therefore, to understand how the radiation dose can be reduced, it is necessary to establish the relationship between image quality and radiation dose.

In CT scanning, image quality has many components and is influenced by many technical parameters. Several metrics describes the different aspects of image quality in CT; noise describes the variation of CT numbers in a physically uniform region. High-contrast spatial resolution, quantifies the minimum size of high-contrast object that can be resolved. Low-contrast spatial resolution quantifies the minimum size of low-contract object that can be differentiated from the background, which is related both to the contrast of the material and the noise-resolution properties of the system. Contrast-to-noise ratio (CNR) and signal-to-noise ratio (SNR) are also some of the common metrics to quantify the overall image quality. ${ }^{15}$ Optimizing the CT system and scanning techniques, improving the image reconstruction and data processing reduces image noise which allows radiation dose reduction.

This article studies the doses and image qualities produced using the default primary scanning factors of our CT machine. Various dose-reduction strategies were used in building scanning protocols that allow radiologists to obtain the necessary diagnostic information while reducing radiation doses to as low as reasonably achievable. 


\section{Methods and Materials}

\section{Dosimetry}

\section{The CT Dose Profiler (CTDP) probe}

The CT Dose Profiler probe is a highly advanced point dose detector that has a solid-state sensor placed $3 \mathrm{~cm}$ from the end of the probe. The probe can be extended with an extension piece made of PMMA to fill different phantoms. The extension is $45 \mathrm{~mm}$. When this is attached, the detector will be centred in the middle of a $150 \mathrm{~mm}$ wide PMMA phantom when the end of the extension reaches the end of the phantom. The sensor is very thin $(250 \mu \mathrm{m})$ in comparison to the beam width and is therefore always completely irradiated when it is in the beam. The sensor collects the dose profile. As radiation hits the sensor, in either direction, the detector registers the dose value at that point and sends the information to the Ocean software. The electrometer can collect 2000 such dose values per second.

When the dose profile is collected, all of the data points are put into a graph. To be able to collect the dose at the different positions, thereby creating the dose profile, the probe must be moved through the CT beam. This is achieved by placing it free in air or in a phantom and then using the couch movement to scan the probe (performs a helical scan). With the CT Dose Profiler you can also see a visible image of the dose profile that will tell you if something is wrong with the system. There is no limit to the slice width that users can measure with the CTDP. When using this probe for CTDI measurements, the traditional five axial scans with an Ion chamber are replaced with one helical (spiral) scan with the CTDP probe in the centre hole of the phantom (head or body). It replaces the conventional thermo luminescent dosimeter (TLD) and optically stimulated luminescence (OSL) methods or film for dose profile measurements. The CT Dose Profiler probe is designed to be used with the Barracuda multimeter and a PC running the Ocean software. ${ }^{16}$

\section{CTDIvol Measurements}

This is the dosimetric quantities employed to characterize dose from the CT scanner, were; the CTDI for a single slice and DLP for a complete or full examination is calculated. CTDI is the most practical quantity that is of concern to the measure in terms of dose during scanning. ${ }^{17}$ There are two ways to perform these measurements. We recommend the use of the central point method were a dedicated CT Dose Profiler Probe and Ocean software is used to perform the measurement and to calculate CTDI values. It is a quick and convenient way to measure all CTDI parameters, DLP, geometrical efficiency and Full Width at Half Maximum
(FWHM). This method is based on the observation that the ratio between $\mathrm{CTDI}_{w}$ and $\mathrm{CTDI}_{100 \text {, central is a constant for spe- }}$ cific CT scanners in combination with the type of phantom used in the measurement. If the k-factor is known, a CTDI100, central measurement is performed, and the RTI Ocean Professional software will then calculate $\mathrm{CTDI}_{\mathrm{w}}$ and CTDIvol automatically. Table 1 gives a list of $\mathrm{k}$-factors supported in the Ocean software for our Siemens CT machine. The Ocean software is used to evaluate and calculate all parameters based on the measured dose profile.

To measure the CTDI 100 with the CT Dose Profiler in the center hole of a head or body phantom with one helical scan and then multiply it with the k-factor to get CTDI ${ }_{w}$ and CTDI vol is, of course faster than doing the five scans with the pencil ion chamber. The probe is either placed inside the phantom via an extension cable or placed in a stand for measurements in air. The measurement was done during a helical (spiral) scan so the table was move during the measurement. Measurements were performed for the most common applied CT examinations covering radiation sensitive organs in the head and body regions. The selected CT examinations used were; head routine, chest routine, abdomen and pelvis routines.

The CT Dose Profiler was connected to the Barracuda via the extension cable and the Barracuda connected to a computer that has the modern Ocean software. The CT head phantom was placed on the head support and the CT Dose Profiler placed in the center hole of the phantom as in Figure 1. The two horizontal CT lasers in the CT room were visible on the probe, approximately in the middle of it. The vertical laser was also approximately in the middle of the phantom. A piece of tape was put along the probe, attaching it onto the phantom to ensure that the probe is not dislodged within the phantom during scanning.

TABLE 1: k-factors for ocean software used for head and body measurement.

\begin{tabular}{cccc}
\hline \hline Manufacturer & $\mathrm{kVp}$ & Head & Body \\
Siemens & 80 & 1.108 & 1.951 \\
Siemens & 110 & 1.055 & 1.666 \\
Siemens & 130 & 1.039 & 1.606 \\
\hline \hline
\end{tabular}

The dose-length product, DLP, includes the irradiated volume and represents the overall exposure for an examination and is calculated as following:

$$
D L P=C T D I_{V O L} \times L
$$

where, $\mathrm{L}=$ scan length of a certain examination. 


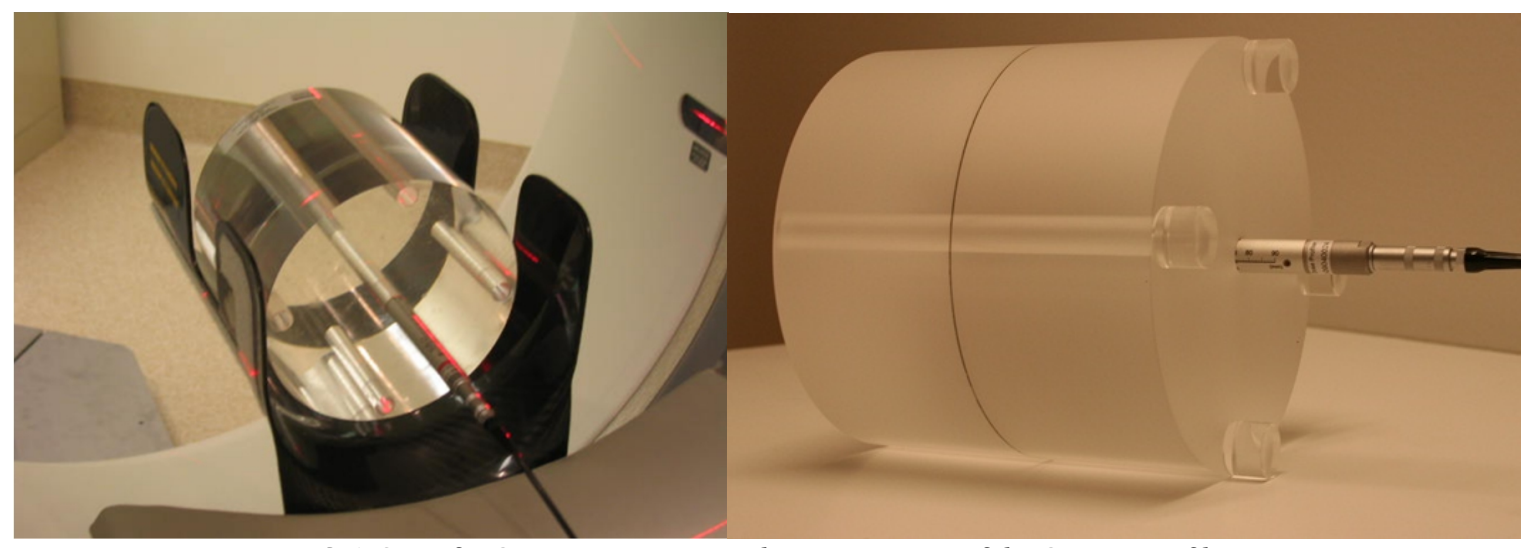

FIG. 1: Setup for CTDI measurement and correct position of the CT Dose Profiler.

\section{Quality Control and Image Quality}

The goal is to ensure that every image created by the CT scanner is of a high quality. High quality images provide the radiologist maximum information, improve the chances for correct diagnosis, and ultimately contribute to quality patient care. There are many QC tests, but in this study, emphasis was given to those tests regarding the relationship between radiation dose and image quality. The most important test in this research was image resolution. Resolution has two components, spatial resolution (high contrast resolution), and contrast resolution (low contrast resolution or detectability). Spatial resolution is the ability to display, as separate images, two objects that are very close to each other. Contrast resolution is the ability to display, as distinct images, areas that differ in density by a small amount. Contrast and spatial resolution are intimately related to each other and to the radiation dose absorbed by the detector.

The Catphan 600 phantom was setted up on the CT couch as shown in Figure 3. The levelling was checked using the spirit level and then aligned using the room lasers. Various scan protocols were used to generate a topogram. These topograms will be used for low and high contrast evaluation and image homogeneity as well.

\section{High contrast evaluation}

In order to check high contrast, set a scanbox or topogram to cover section CTP528, as in Figure 3. Reconstruct the images using $1 \mathrm{~mm}$ slice thickness and evaluate the contrast. Choose a section in the region were the line pairs are fully visible and read the number of clearly separated line pairs detectable by the eye without zooming the image. Adjust the window settings to obtain an optimal image prior to read out.

\section{Low contrast evaluation}

Position a scanbox or topogram over section CTP515 which contains the low contrast targets as seen in Figure 3. To determine the actual contrast levels, average measurements were made over from several scans. It is important to measure the background area adjacent to the measured target.
The ROI should be at least $4 \times 4$ pixels in diameter to eliminate noise. Reconstruct image sets using $10 \mathrm{~mm}$ slice thickness, position the ROI tool on the large discs, and make notes of the Hounsfield units (HU) and Standard deviations (SD). Furthermore, position ROI tool close to the discs for a background measurement of $\mathrm{HU}$ and SD.

\section{Results}

The default factory scanning parameters of our Siemens CT with model Somatom Emotion (16 channel detector) for the various examinations are as follows: The head sequence scans are done by means of the conventional (axial) technique, using $130 \mathrm{kVp}$, an $8 \mathrm{~mm}$ slice thickness for the cerebrum examination and $5 \mathrm{~mm}$ for the skull base. A tube voltage of $130 \mathrm{kVp}$ and a $5 \mathrm{~mm}$ slice thicknesses are used for trunk examinations (thorax, abdomen and pelvis). Regarding the kilovoltage selection, in general the range was between 80-130 KV and $130 \mathrm{KV}$ was the preferred in most cases because it results in good image quality without excessive tube load.

Table 2 shows the standard default parameters used in performing the different examinations of this study. These parameters were used by the operators (radiographers) for average weight adult patients $(60.9 \mathrm{~kg})$. The variable parameter was the mAs setting, which ranged between $240-270 \mathrm{mAs}$ for head exams protocols, and 100-120 mAs for the trunk exams protocols.

TABLE 2: Default scanning parameters used for the four (4) different common CT examinations. (Adults)

\begin{tabular}{|c|c|c|c|c|}
\hline Examination & $\mathrm{kV}$ & $\mathrm{mAs}$ & $\begin{array}{l}\text { No. of } \\
\text { Slices }\end{array}$ & $\begin{array}{l}\text { Slice thickness } \\
(\mathrm{mm})\end{array}$ \\
\hline \multicolumn{5}{|l|}{ Head Sequence } \\
\hline Cerebrum & 130 & 270 & 12 & 8 \\
\hline Base & 130 & 270 & 8 & 5 \\
\hline Thorax routine & 130 & 100 & 62 & 5 \\
\hline Abdomen routine & 130 & 120 & 40 & 5 \\
\hline Pelvic routine & 130 & 120 & 40 & 5 \\
\hline
\end{tabular}




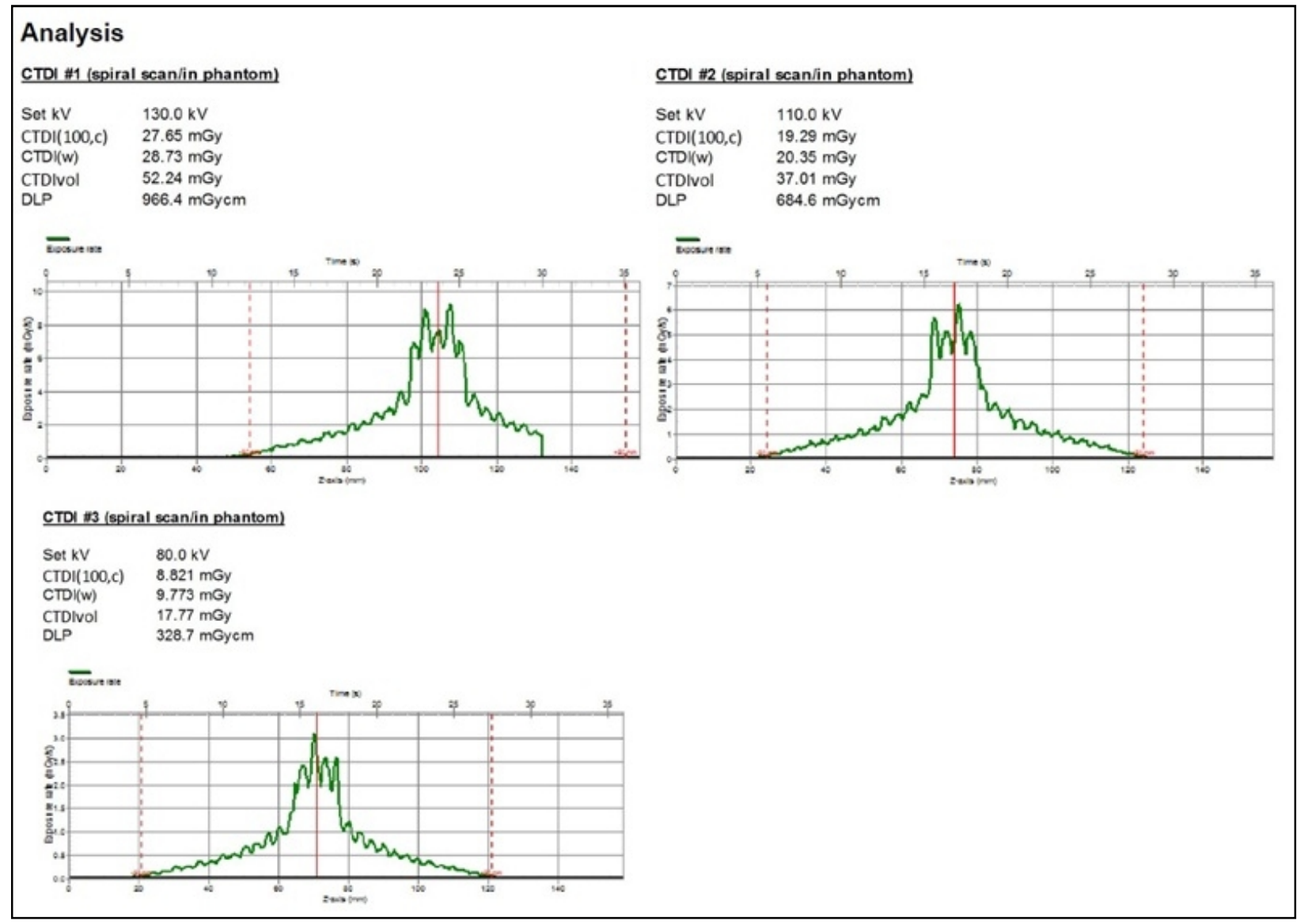

FIG. 2: Analysis using the CT Dose Profiler Probe and Ocean software for measurements.

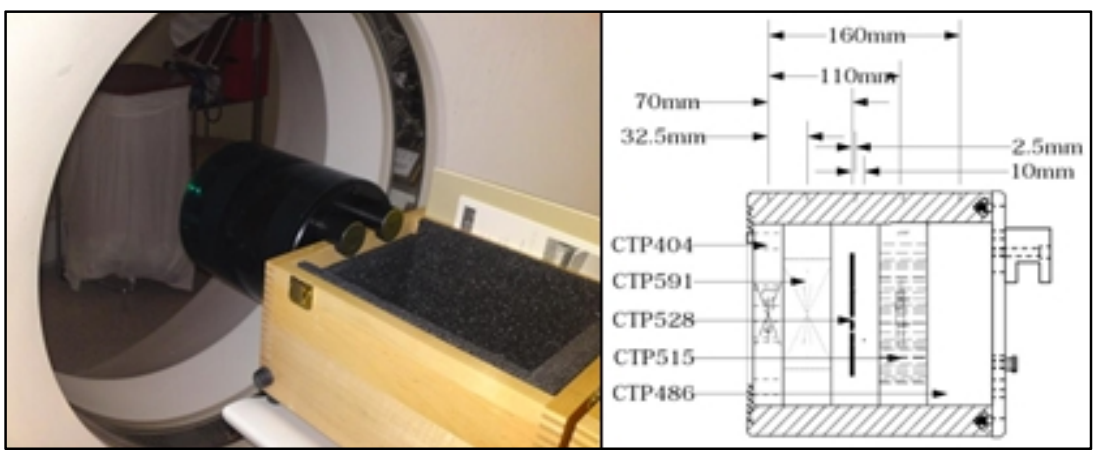

FIG. 3: Catphan measurement set-up and sections usedSection CTP515 of the Catphan contains the low contrast module with supra-slice and sub-slice contrast targets as in Figure 4. Using the ROI (region of interest), positioned on the large discs, all Hounsfield unit and standard deviation were made.

TABLE 3: Measurements of CTDI and DLP for the head phantom.

\begin{tabular}{ccc}
\hline \hline Kilovolt (KV) & CTDIvol (mGy) & DLP (mGy.cm) \\
130 & 52.24 & 966.4 \\
110 & 37.01 & 684.6 \\
80 & 17.77 & 328.7 \\
\hline \hline
\end{tabular}

Table 3 gives the CTDI and DLP values at varying KV parameter with all other parameters held constant. From Table 3 , reducing the kilovoltage from 130 to $80 \mathrm{KV}$ leads to a $66 \%$ decrease in radiation dose at a constant $\mathrm{mAs}$ setting because the dose varies with the square of the kilovoltage. Table 5 below also confirms the effect of varying KV with dose. This reduction correlates with increased image noise and potentially with decreased image quality. Thus, this voltage reduction should be compensated by increasing the tube current. ${ }^{18}$

CTDI and DLP values are indicators of the local dose in the irradiated slice and the total radiation exposure to the patient 
respectively. They are used to evaluate dose parameters and compare performance against reference criteria.

TABLE 4: Average CTDI and DLP values for various patient exams compared with EC reference values (adults).

\begin{tabular}{ccc}
\hline \hline Examination & CTDI Ivol $_{(\mathrm{mGy})}$ & DLP $(\mathrm{mGy} . \mathrm{cm})$ \\
Head Sequence & & \\
Cerebrum & 57.54 & 624.28 \\
Base & 58.32 & 874.53 \\
Thorax routine & 7.88 & 381.79 \\
Abdomen routine & 12.41 & 559.12 \\
Pelvic routine & 11.44 & 305.03 \\
EC Reference Dose & & \\
Head & 60 & 1050 \\
Chest & 30 & 650 \\
Abdomen & 35 & 800 \\
Pelvis & 35 & 600 \\
\hline \hline
\end{tabular}

The average CTDI ${ }_{\text {vol }}$ and DLP values for different patient examinations using the build-up protocols are given in Table 4 along with the European Commission (EC) reference levels. There are no examinations with values above the EC reference levels.

The most important part of the quality control and image quality procedures are the determination of high and low resolution of the CT image. Spatial resolution (high contrast resolution) describes the degree of blurring in an image; that is a measure of the ability to discriminate objects of varying density a small distance apart against a uniform background. The Low contrast resolution is the ability of the CT scanner to demonstrate small changes in tissue contrast. The effect of selecting tube $\mathrm{KV}$ and $\mathrm{mAs}$ on both spatial resolution and low contrast were examined for three $\mathrm{KV}$ values $(80,110$ and 130); the mAs values were degraded from between 50 to 300 mAs.

For high contrast resolution, section CTP714 of the CT Catphan phantom, see Figure $\mathbf{3}$ is scanned and the images evaluated. Most of the line pairs chosen were fully visible to read by the eye without zooming the images. Window settings were adjusted to obtain an optimal image prior to read out. All low contrast pins and spatial resolution pins were clearly resolved in optimum mAs and KV settings (130 KV and 240 mAs or higher).

Figure 5 shows the image quality of the QC process in terms of high resolution and low contrast. In order to be assured of these image quality results obtained by the $\mathrm{QC}$ phantom, a head Rando anthropomorphic phantom (representing an average sized human head) was used to achieve good image quality. The phantom has almost the same densities of the human head components of bone and soft tissue materials. The head phantom was scanned with the new head protocol with varying window level settings as shown in Figure 6. For the cerebrum window level settings, although image quality increases at higher $\mathrm{kV}$ and $\mathrm{mAs}$ settings, images at lower settings were also at acceptable level of quality.

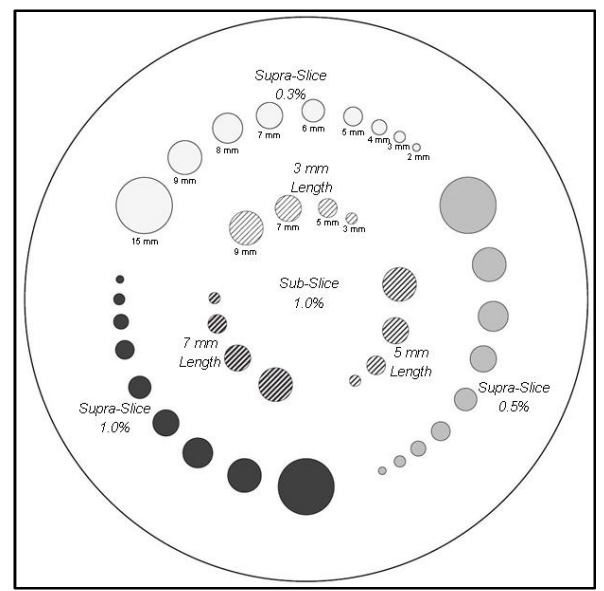

FIG. 4: Catphan CTP515 low contrast module.

\section{Discussion}

The CTDI values using the default scanning parameters were comparable with international recognized values for the truck examinations. Whiles the CTDI values for the head examinations were in most cases a bit higher than the refer-

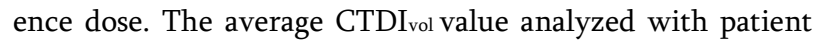
scans was $65.45 \mathrm{mGy}$ as compared to $60 \mathrm{mGy}$ for the head by EC reference value. The DLP for the head examinations were also observed to be higher by an average factor of 3.4, hence the need to optimize these parameters to reduce patient radiation doses. The new protocol was able to drastically reduce the DLP values of the head protocol from an average value of $2279.85 \mathrm{mGy} . \mathrm{cm}$ to an average of $874.53 \mathrm{mGy} . \mathrm{cm}$. This was done not to degrade the quality of the images produced but a delicate balance between the two parameters (dose and image quality). Dosimetry and QC measurements were conducted with various equipment and phantoms to build new scanning protocols.

From the results, when the kilovolt peak was increased from 110 to 130 , the CTDIvol increase was $41 \%$ in the head phantom. From Figure 2, the analysis using the CT Dose Profiler Probe and Ocean software showed direct proportional correlation between the KV and CTDI/DLP values. When all technical parameters are held constant and the KV is increased, the dose value is also increased for both the head and body dosimetry phantoms. The radiation dose is also linear with the mAs values when all other factors are held constant. So if the mAs value is reduced by $50 \%$, the radiation dose will be reduced by the same amount.

However, this reduction increase image noise by this relation (i.e. $1 \sqrt{ } \mathrm{mAs}$ ), which means that a $50 \%$ reduction in the $\mathrm{mAs}$ value will results in a noise increase of $41 \%$. Depending on the requirements of the clinical application, this reduc- 
tion of $50 \%$ in dose resulting in a $41 \%$ increment in image noise may readily be accepted; because this type of reduction will compromise the diagnostic quality of the examination. Detection of high-contrast objects in the thorax region (e.g. lung) may not require a low-noise imaging protocol and hence the reduction in $\mathrm{mAs}$ (i.e. less dose to lung) will be well tolerated. On the other hand, imaging low-contrast lesions in the abdomen region (e.g. liver) does require a low-noise imaging protocol and hence the reduction in $\mathrm{mAs}$ will limit the ability to detect these lesions.

The 30 line pair per $\mathrm{cm}$ gauge resolution tests for visual evaluation of the high resolution as shown in Figure 5 gave better resolution. In this study, majority of line pairs per $\mathrm{cm}$ gauge of spatial resolution pins were resolved in most cases.

TABLE 5: Measurements performed with the head phantom and Ocean software using different KV values.

\begin{tabular}{|c|c|c|c|c|c|c|c|c|c|}
\hline \# & $\begin{array}{c}\text { Set kV } \\
(\mathrm{kV})\end{array}$ & $\begin{array}{c}\text { CT Phantom } \\
\text { type }\end{array}$ & $\begin{array}{l}\text { Collimation } \\
(\mathrm{mm})\end{array}$ & Pitch & $\begin{array}{l}\text { Scan length } \\
(\mathrm{mm})\end{array}$ & $\begin{array}{l}\text { Tube rota- } \\
\text { tion time (s) }\end{array}$ & $\begin{array}{c}\text { Scan speed } \\
(\mathrm{mm} / \mathrm{s})\end{array}$ & $\begin{array}{c}\text { Measuring } \\
\text { time (s) }\end{array}$ & $\begin{array}{c}\text { Exposure } \\
\text { (mGy) }\end{array}$ \\
\hline 1 & 130.0 & Head & 12 & 0.550 & 185 & 1.50 & 4.40 & 30 & 51.36 \\
\hline 2 & 110.0 & Head & 12 & 0.550 & 185 & 1.50 & 4.40 & 30 & 36.28 \\
\hline 3 & 80.0 & Head & 12 & 0.550 & 185 & 1.50 & 4.40 & 30 & 16.32 \\
\hline
\end{tabular}

${ }^{*}$ note that Exposure as calculated by the Ocean software in Table 5 refers to the dose.

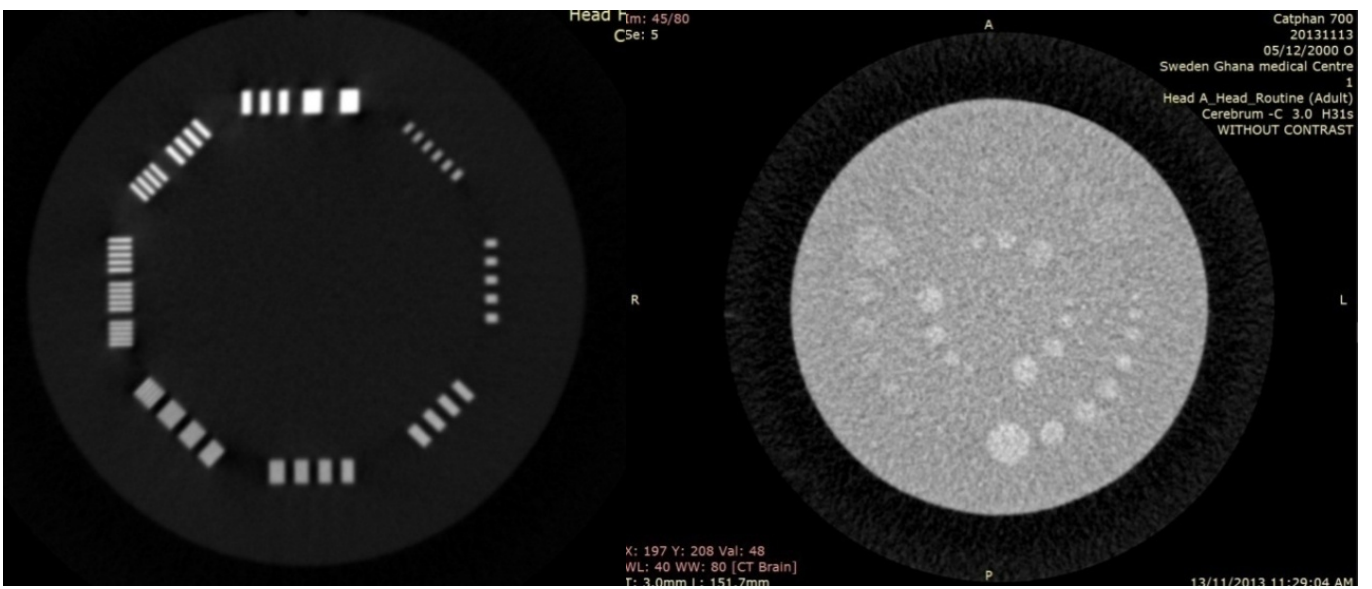

FIG. 5: High resolution and low contrast modules of the QC procedure.

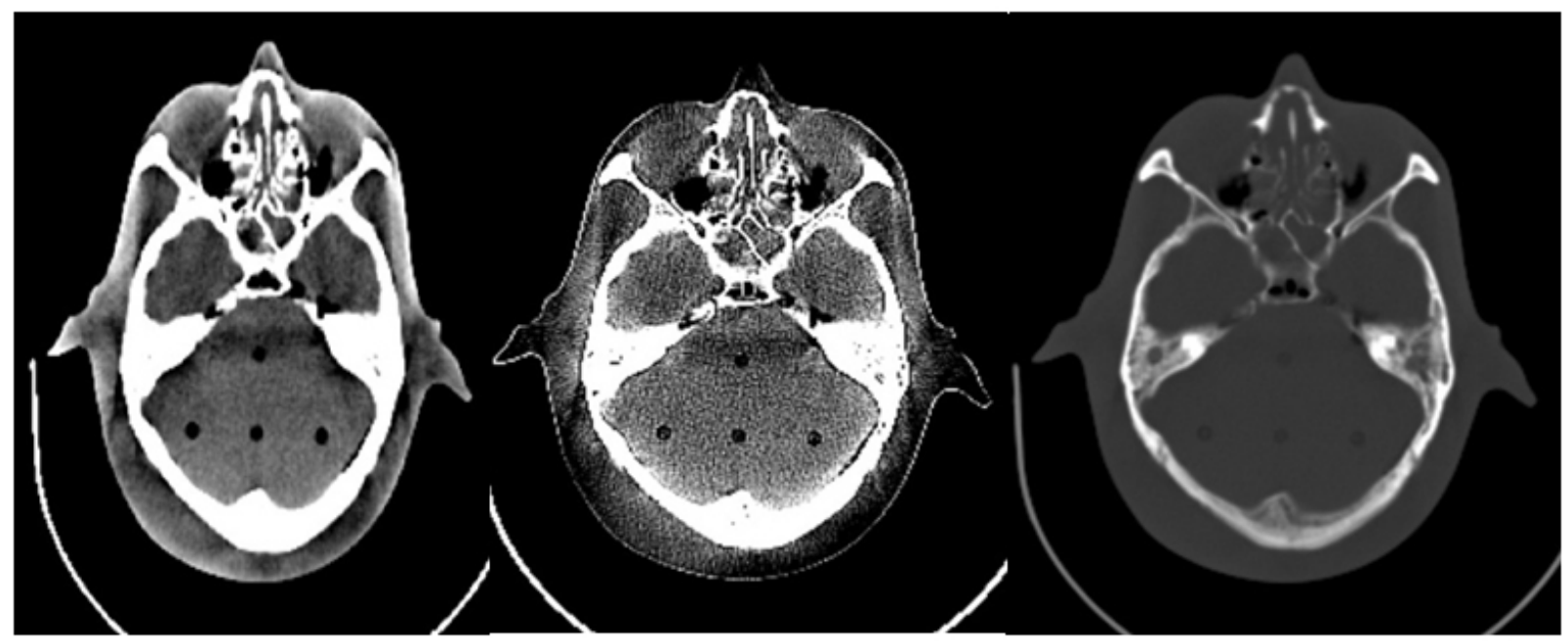

FIG. 6: Images of the skull using different windows and settings. 
The actual low contrasts levels were measured by making region of interest measurements over the larger targets, and in the local background area. The HU values measured had very close values for each range of target and the background measurement (typically from 4-10 HU difference). This confirmed the ability to display, as distinct images, areas that differ in density by a small amount.

Apart from the KV and mAs selections which affect both image quality and patient radiation dose, the spatial resolution and low contrast, were also enhanced by using reconstruction algorithm selection (software filter) and the FOV (field of view). FOV determine the diameter of the reconstructed image, smaller FOV reduces the pixel size and hence improve spatial resolution. The filter parameter is used to set the mathematical algorithm which determines the sharpness or smoothness of the image. Noise in image increases as sharpness of the image increases, and vice versa. In general, low contrast decreases as spatial resolution and noise increases. There are wide selections of kernels and windows in modern CT scanners, which can give wide range of image contrast and resolution. There are different kernels also assigned to medium, sharp, low resolution and high resolution for different parts of the body.

In general, the selection of physical factors, such as $\mathrm{KV}$, mAs and slice thickness, had a direct influence on patient radiation dose. However those factors also affected image quality. Increasing exposure increases low contrast resolution by reducing noise but also increases patient dose. Image quality consistent with the clinical indications was achieved with the lowest possible dose to the patient.

\section{Conclusion}

In conclusion, results of image quality of this study are encouraging to select lower parameters for general cases where higher resolution images are not intended. A careful minimization in scanning factors ( $\mathrm{KV}$ and $\mathrm{mAs}$ ), especially for children and thinner adult patients, are achieved in order to attain optimum degree of image quality and radiation dose saving. Determination of imaging parameters is the responsibility of medical staff (Medical physicists, Radiographers and engineers), according to the machine performance and diagnosis requirements. On the basis of this study, the diagnostic department at Sweden Ghana Medical Centre is still carrying out extended studies, with the cooperation of radiologists, technicians and medical physicists to continue to investigate all factors which affect patient radiation dose and image quality, in order to ensure optimum level of radiological diagnosis. The practice should ensure that patient doses are kept as low as reasonably achievable. This process of radiation dose optimization with scanning parameter selection must begin with making age-specific and size-specific pro- tocols, but must continue onward, tailoring radiation doses according to specific clinical indications in each body region.

Methods to reduce radiation dose may involve tradeoffs in image quality, and many times the different image quality characteristics are inter-related. The more clearly defined the objectives of a clinically indicated study, then the more clearly the image quality requirements can be determined.

\section{Conflict of interest}

The authors declare that they have no conflicts of interest. The authors alone are responsible for the content and writing of the paper.

\section{Acknowledgements}

This manuscript is based on work done at the Sweden Ghana Medical Centre, Accra, Ghana from January 2012 to January 2014. We wish to express our gratitude to the oncologists, physicists, dosimetrist, therapists, radiographers, engineer, and other members of the treatment team and all staff of SGMC. This work is also dedicated to the memory of the late Mr. Emmanuel Kwaku Nani (a pioneer of Medical Physics in Ghana, a mentor and a great friend).

\section{References}

1. United Nations Scientific Committee on the Effects of Atomic Radiation (UNSCEAR). Sources and effects of ionizing radiation. Volume 1, Annexe A: Medical radiation exposures. Report to the General Assembly with Scientific Annexes. New York: UnitedNations, 2008. Available from http://www.unscear.org/docs/reports/2008/09-8675 3_Report_2008_GA_Report_corr2.pdf

2. Biologic effects of ionizing radiation (BEIR) report VII. Health risks from exposure to low levels of ionizing radiation, National Academies, 2006. http://www.dep.state.pa.us/brp/radon_division/BEI R\%20VII\%20Preliminary\%20Report.pdf

3. McCollough CH, Primak AN, Branu N, et al. Strategies for reducing radiation dose in CT. RadiolClin North Am 2009; 47: 27-40.

4. The Royal College of Radiologists: Making the best use of clinical radiology services: referral guidelines. $6^{\text {th }}$ Edition, London, 2007.

5. ICRP. 1990 Recommendations of the International Commission on Radiological Protection. ICRP Publications 60. Ann. ICRP 21, Pergamon Press, Oxford; 1991.

6. Sources and effects of ionizing radiation, Unscear 2000. New York: United Nations, 2000; 1:304-5. 
7. Hart D, Wall BF. UK population dose from medical X-ray examinations. EurJRadiol 2004; 50:285-91.

8. Department of Health. Form KH12: Number of imaging and radio diagnostic examinations or tests, NHS organization in England, 2003-2004.

9. Clarke J, Cranley K, Robinson J, et al. Application of draft European commission reference levels to a regional CT dose survey. Br J Radiol 2000; 73:43-50.

10. Wade JP, Weyman JC, Goldstone KE. CT standard protocols are of limiting value in assessing actual patient dose. Br J Radiol 1997; 70: 1146-51.

11. AAPM. The measurement, reporting and management of radiation dose in CT. Report of AAPM Task Group 23 of the diagnostic imaging council CT committee. College Park, MD: AAPM; 2008.

12. Descamps C, Gonzalez M, Garigo E, et al. Measurements of dose delivered during CT exams using AAPM Task Group Report No. 111. J ApplClin Med Phys 2012; 13:3934.

13. EUR16262 EN. European guidelines on quality criteria for computed tomography. European Commission, Luxembourg, 2000.
14. American College of Radiology. ACR practice guideline for diagnostic reference levels in medical $\mathrm{x}$-ray imaging. In: Practice guidelines and technical standards. American College of Radiology, Reston. VA, USA. 2008; 799-804.

15. Callstrom MR, Johnson CD, Fletcher JG, et al. CT colonography without cathartic preparation: feasibility study. Radiology 2001; 219: 693-8.

16. www.rti.se

17. Shope TB, Gagne RM, Johnson GC. A method for describing the doses delivered by transmission X-ray computed tomography. Med Phys 1981; 8:488-95.

18. Sigal-Cinqualbre $\mathrm{AB}$, Hennequin $\mathrm{R}$, Abada HT, et al. Low-Kilovoltage Multi-detector row chest CT in adults: feasibility and effect on image quality and iodine dose. Radiology 2004; 231:169-74. 